\title{
Modèle d'évolution de la croissance de boeufs Ndama exploitant les repousses après feux en savane de la zone guinéenne au Zaïre (Ranch de Kolo)
}

\author{
Ph Lecomte 1, P Frère 2, JF Renard 2, R Oger 1 \\ ${ }^{1}$ CRAG Station de Haute Belgique B6800 Libramont; ${ }^{2}$ Compagnie J Van Lancker, \\ B1050 Bruxelles, Belgique
}

\begin{abstract}
En élevage extensif de bovins sur savane naturelle de la zone guinéenne, le croît des animaux varie au cours de l'année, selon les saisons. Au ranch de Kolo, situé au bas Zaïre, la pluviosité annuelle est de $1300 \mathrm{~mm}$, la saison sèche s'étend de mai à septembre et l'on observe un ralentissement des pluies en février (petite saison sèche). Le bétail Ndama exploite les repousses de graminées naturelles qui font suite aux feux précoces, tardifs et de contresaison. Dans chaque parc occupé par un troupeau, l'éleveur exploite en rotation 4 parcelles dont une reste en défens annuel, les feux étant organisés respectivement pour la région du basZaïre, au cours des mois de juin, septembre et février.
\end{abstract}

Afin de caractériser l'évolution du poids du bétail Ndama en conditions d'élevage extensif de savane, des pesées systématiques ont été effectuées au cours de deux années au ranch de Kolo sur des boeufs âgés de 12 à 54 mois. Les animaux différenciés selon le mois de naissance ont été pesés à 4 époques caractéristiques du climat régional : février, petite saison sèche ; mai, fin de la deuxième période de pluies - début de la saison sèche ; septembre, fin de la saison sèche; novembre, pleine saison des pluies.

Pour permettre de décrire en continu l'évolution théorique du poids d'un animal né à une période donnée, l'ensemble des données individuelles récoltées a été traité dans un modèle à 2 composantes basé sur les hypothèses suivantes:

- D'une part, entre 12 et 54 mois, la courbe générale de poids à une période donnée correspond à une sigmoïde asymétrique $y=M /\left((1+n * \exp ((-x-a) / b))^{1 / n}\right)$, avec «y» le poids à une période donnée, "M» le poids maximum, " $n$ " le paramètre de dissymétrie de la courbe, " $X$ " l'âge de l'animal, «a» l'abscisse de la croissance la plus élevée et «b» la vitesse de croissance. Le modèle, de type Nelder (1962, Biometrics, 18 , 614-616) est similaire à celui utilisé par Ahunu et al (1994, Discovery and Innovation, 6(1), 78-83) pour décrire les poids selon l'âge du bétail
Ndama au Ghana.

- D'autre part, la variation saisonnière de poids peut être décrite en assortissant cette première composante d'une fonction trigonométrique de type $\mathrm{K} \sin (\mathrm{Wi}+\varphi)$ où l'amplitude $\mathrm{K}$ varie en fonction du poids de l'animal. Wi $(2 \pi \mathrm{i} / 12)$ étant la compo-sante cyclique annuelle (i étant le mois de l'année) et $\varphi$ un facteur de déphasage de la fonction trigonométrique qui implicitement traduit le décalage de la sinusoïde par rapport aux solstices.

2329 données ont été traitées (procédure de régression non linéaire SAS). Pour l'ensemble des valeurs, l'ajustement final est caractérisé, par un coefficient de détermination $\mathrm{R}^{2}=0,82$ et un écart-type résiduel de $21,2 \mathrm{~kg}$. Si l'on s'en tient aux valeurs moyennes par mois de naissance, la régression entre les valeurs prédites et celles observées est caractérisée par un $\mathrm{R}^{2}$ de 0,95 et un écart-type résiduel de $12,1 \mathrm{~kg}$.

Sur un plan pratique le modèle constitue un référentiel technique utilisable pour planifier ou simuler des productivités pondérales annuelles et pour tester différentes stratégies en matière d'âge d'abattage selon les effectifs et la répartition des naissances au cours de l'année. II peut être utile pour le calcul de charges animales lors de la conception de programmes de développpement d'élevages. Sur le terrain, il permet la comparaison des poids de lots d'animaux à ce que l'on devrait observer en situation normale d'élevage extensif et éventuellement, l'estimation des niveaux de complémentation à pourvoir afin de minimiser les pertes de poids. Le modèle (théorique) ne peut actuellement prendre en compte les importantes variations inter-annuelles de durée de la saison sèche. Il pourrait être complété sur cet aspect dans la mesure où l'on disposerait de données réparties sur un plus grand nombre d'années climatiquement distinctes. Sous d'autres latitudes, la position et l'amplitude de la saison sèche étant différentes, le modèle devra être adapté. A ce stade de la démarche, il n'est valable que pour une situation moyenne au bas Zaïre.

$\begin{array}{lcccccccc}\text { Paramètres } & \mathrm{M} & \mathrm{b} & \mathrm{a} & \mathrm{n} & \mathbf{k} & \varphi & \mathrm{R}^{2} & \text { etr } \\ \text { Valeur } & 332 & 8,07 & 27,26 & 3,96 & 0,0822 & -0,1624 & 0,82 & 21,2 \\ \text { écart type } & 12,6 & 1,93 & 1,41 & 1,21 & 0,003 & 0,044 & & \end{array}$

